\title{
BMO and Lipschitz norm estimates for the composition of Green's operator and the potential operator
}

\author{
Zhimin Dai ${ }^{1}$, Yuming Xing ${ }^{1 *}$ and Shusen Ding ${ }^{2}$
}

\section{${ }^{*}$ Correspondence:}

xyuming@hit.edu.cn

'Department of Mathematics,

Harbin Institute of Technology,

Harbin, China

Full list of author information is

available at the end of the article

\begin{abstract}
In this paper, we establish BMO and Lipschitz norm inequalities for the composition of Green's operator and the potential operator. We also investigate the relationship among the Lipschitz norm, the BMO norm and the $L^{p}$-norm. Finally, we display some examples for applications.

MSC: Primary 35J60; secondary 31B05; 58A10; 46E35

Keywords: differential forms; Lipschitz norm; BMO norm; Green's operator; potential operator
\end{abstract}

\section{Introduction}

Differential forms are extensions of functions and can be used to describe various systems in partial differential equations (or PDEs), physics, theory of elasticity, quasiconformal analysis, etc. Differential forms have become invaluable tools for many fields of sciences and engineering; see [1,2] for more details.

Now we introduce some notations and definitions. Let $\Theta$ be an open subset of $\mathbb{R}^{n}(n \geq 2)$ and $O$ be a ball in $\mathbb{R}^{n}$. Let $\rho O$ denote the ball with the same center as $O$ and $\operatorname{diam}(\rho O)=$ $\rho \operatorname{diam}(O), \rho>0$. A weight $w(x)$ is a nonnegative locally integrable function in $\mathbb{R}^{n} .|D|$ is used to denote the Lebesgue measure of a set $D \subset \mathbb{R}^{n}$. Let $\wedge^{\ell}=\wedge^{\ell}\left(\mathbb{R}^{n}\right), \ell=0,1, \ldots, n$, be the linear space of all $\ell$-forms $\hbar(x)=\sum_{J} \hbar_{J}(x) d x_{J}=\sum_{J} \hbar_{j_{1} j_{2} \cdots j_{\ell}}(x) d x_{j_{1}} \wedge d x_{j_{2}} \cdots \wedge d x_{j_{\ell}}$ in $\mathbb{R}^{n}$, where $J=\left(j_{1}, j_{2}, \ldots, j_{\ell}\right), 1 \leq j_{1}<j_{2}<\cdots<j_{\ell} \leq n$, are the ordered $\ell$-tuples. Moreover, if each of the coefficient $\hbar_{J}(x)$ of $\hbar(x)$ is differential on $\Theta$, then we call $\hbar(x)$ a differential $\ell$-form on $\Theta$ and use $D^{\prime}\left(\Theta, \wedge^{\ell}\right)$ to denote the space of all differential $\ell$-forms on $\Theta . C^{\infty}\left(\Theta, \wedge^{\ell}\right)$ denotes the space of smooth $\ell$-forms on $\Theta$. We denote the exterior derivative by $d$, and the Hodge codifferential operator $d^{\star}$ is defined as $d^{\star}=(-1)^{n \ell+1} \star d \star: D^{\prime}\left(\Theta, \Lambda^{\ell+1}\right) \rightarrow D^{\prime}\left(\Theta, \Lambda^{\ell}\right)$, where $\star$ is the Hodge star operator. For $1 \leq p<\infty, L^{p}\left(\Theta, \wedge^{\ell}\right)$ is a Banach space with the norm $\|\hbar\|_{p, \Theta}=\left(\int_{\Theta}|\hbar(x)|^{p} d x\right)^{1 / p}=\left(\int_{\Theta}\left(\sum_{J}\left|\hbar_{J}(x)\right|^{2}\right)^{p / 2} d x\right)^{1 / p}<\infty$. For a weight $w(x)$, we write $\|\hbar\|_{p, \Theta, w}=\left(\int_{\Theta}|\hbar|^{p} w(x) d x\right)^{1 / p}$. Similarly, the notations $L_{\text {loc }}^{p}\left(\Theta, \wedge^{\ell}\right)$ and $W_{\text {loc }}^{1, p}\left(\Theta, \wedge^{\ell}\right)$ are self-explanatory.

From [3], if $\hbar$ is a differential form in a bounded convex domain $\Theta$, then there is a decomposition

$$
\hbar=d(T \hbar)+T(d \hbar)
$$

\section{Springer}

(0) 2013 Dai et al.; licensee Springer. This is an Open Access article distributed under the terms of the Creative Commons Attribution License (http://creativecommons.org/licenses/by/2.0), which permits unrestricted use, distribution, and reproduction in any medium, provided the original work is properly cited. 
where $T$ is called a homotopy operator. For the homotopy operator, we know that

$$
\|T \hbar\|_{p, O} \leq C|O| \operatorname{diam}(O)\|\hbar\|_{p, O}
$$

holds for any differential form $\hbar \in L_{\mathrm{loc}}^{p}\left(O, \wedge^{\ell}\right), \ell=1,2, \ldots, n, 1<p<\infty$. Furthermore, we can define the $\ell$-form $\hbar_{\Theta} \in D^{\prime}\left(\Theta, \wedge^{\ell}\right)$ by

$$
\hbar_{\Theta}= \begin{cases}|\Theta|^{-1} \int_{\Theta} \hbar(y) d y, & \ell=0 \\ d(T \hbar), & \ell=1, \ldots, n\end{cases}
$$

for all $\hbar \in L^{p}\left(\Theta, \wedge^{\ell}\right), 1 \leq p<\infty$.

In this paper, we focus on a class of differential forms satisfying the well-known nonhomogeneous A-harmonic equation

$$
d^{\star} A(x, d \hbar)=B(x, d \hbar)
$$

where $A: \Theta \times \wedge^{\ell}\left(\mathbb{R}^{n}\right) \rightarrow \wedge^{\ell}\left(\mathbb{R}^{n}\right)$ and $B: \Theta \times \wedge^{\ell}\left(\mathbb{R}^{n}\right) \rightarrow \wedge^{\ell-1}\left(\mathbb{R}^{n}\right)$ satisfy the conditions: $|A(x, \eta)| \leq a|\eta|^{s-1}, A(x, \eta) \cdot \eta \geq|\eta|^{s}$ and $|B(x, \eta)| \leq b|\eta|^{s-1}$ for almost every $x \in \Theta$ and all $\eta \in \wedge^{\ell}\left(\mathbb{R}^{n}\right)$. Here $a, b>0$ are some constants and $1<s<\infty$ is a fixed exponent associated with (1.4). A solution to (1.4) is an element of the Sobolev space $W_{\mathrm{loc}}^{1, s}\left(\Theta, \wedge^{\ell-1}\right)$ such that

$$
\int_{\Theta} A(x, d \hbar) \cdot d \psi+B(x, d \hbar) \cdot \psi=0
$$

for all $\psi \in W_{\text {loc }}^{1, s}\left(\Theta, \wedge^{\ell-1}\right)$ with compact support. The various deformations of (1.4) are shown in [1].

Recently, Bi extended the definition of a potential operator to the set of all differential forms in [4]. For any differential $\ell$-form $\hbar(x)=\sum_{J} \hbar_{J}(x) d x_{J}$, the potential operator $P$ is defined by

$$
P \hbar(x)=P\left(\sum_{J} \hbar_{J}(x) d x_{J}\right)=\sum_{J} P\left(\hbar_{J}(x)\right) d x_{J}=\sum_{J} \int_{\Theta} K(x, y) \hbar_{J}(y) d y d x_{J}
$$

where the kernel $K(x, y)$ is a non-negative measurable function defined for $x \neq y, \hbar_{J}(x)$ is defined on $\Theta \subset \mathbb{R}^{n}$ and the summation is over all ordered $\ell$-tuples $J$. For more results related to the potential operator $P$, see [4-6].

Green's operator and the potential operator are of quite importance in the study of potential theory and nonlinear elasticity; see [1, 2, 4, 7-10] for more properties of these two operators. In many situations, the process of studying solutions of PDEs involves estimating the various norms of the operators. However, the study on the composition of the potential operator and other operators is yet to be fully developed. Hence, we are motivated to establish some norm inequalities for the composite operator $G \circ P$ applied to differential forms.

It is well known that Lipschitz and BMO norms are two kinds of important norms in differential forms, which can be found in [11]. Now we recall these definitions as follows. 
Let $\hbar \in L_{\text {loc }}^{1}\left(\Theta, \wedge^{\ell}\right), \ell=0,1, \ldots, n$. We write $\hbar \in \operatorname{locLip}_{k}\left(\Theta, \wedge^{\ell}\right), 0 \leq k \leq 1$, if

$$
\|\hbar\|_{\operatorname{locLip}_{k}, \Theta}=\sup _{\rho O \subset \Theta}|O|^{-(n+k) / n}\left\|\hbar-\hbar_{O}\right\|_{1, O}<\infty
$$

for some $\rho \geq 1$. Further, we write $\operatorname{Lip}_{k}\left(\Theta, \wedge^{\ell}\right)$ for those forms whose coefficients are in the usual Lipschitz space with exponent $k$ and write $\|\hbar\|_{\operatorname{Lip}_{k}, \Theta}$ for this norm. Similarly, for $\hbar \in L_{\mathrm{loc}}^{1}\left(\Theta, \wedge^{\ell}\right), \ell=0,1, \ldots, n$, we write $\hbar \in B M O\left(\Theta, \wedge^{\ell}\right)$ if

$$
\|\hbar\|_{\star, \Theta}=\sup _{\rho O \subset \Theta}|O|^{-1}\left\|\hbar-\hbar_{O}\right\|_{1, O}<\infty
$$

for some $\rho \geq 1$. When $\hbar$ is a 0 -form, equation (1.8) reduces to the classical definition of $B M O(\Theta)$. As to the definitions of the weighted Lipschitz and BMO norms, we will present them in Section 3.

The purpose of this paper is to derive the Lipschitz and BMO norm inequalities for the composition of Green's operator $G$ and the potential operator $P$ applied to differential forms.

\section{Estimates for Lipschitz and BMO norms}

In this section, we establish the estimates for Lipschitz and BMO norms for the composite operator $G \circ P$. We need the following lemmas and definition.

The following inequality is the well-known Hölder inequality and gets proved with the Cauchy-Schwarz inequality in [12].

Lemma 2.1 Let $(\Theta, \mu)$ be a measure space and $L^{p}(\mu)=L^{p}(\Theta, \mu)=\left\{f: \Theta \subset \mathbb{R}^{n} \rightarrow\right.$ $\left.\mathbb{C} ;\|f\|_{p, \Theta, \mu}<\infty\right\}$ be a Lebesgue space with the $L^{p}$-norm

$$
\|f\|_{p, \Theta, \mu}= \begin{cases}\left(\int_{\Theta}\|f\|^{p} d \mu\right)^{1 / p}, & 1 \leq p<\infty ; \\ {\operatorname{ess} \sup _{x \in \Theta}|f(x)|,} p=\infty .\end{cases}
$$

If $p, q \geq 1$ with $1 / p+1 / q=1$, and iff $\in L^{p}(\mu)$ and $g \in L^{q}(\mu)$, then $f g \in L^{1}(\mu)$ and

$$
\|f g\|_{1, \Theta, \mu} \leq\|f\|_{p, \Theta, \mu}\|g\|_{q, \Theta, \mu} .
$$

Remark If $\mu$ is a Lebesgue measure, that is, $d \mu=d x$, then (2.2) reduces to the inequality

$$
\|f g\|_{1, \Theta} \leq\|f\|_{p, \Theta}\|g\|_{q, \Theta} .
$$

Lemma 2.2 [11] Let $\hbar \in D^{\prime}\left(\Theta, \wedge^{\ell}\right)$ be a solution to the nonhomogeneous $A$-harmonic equation (1.4) on $\Theta$ and $\rho>1$ be a constant. Then there exists a constant $C$, independent of $\hbar$, such that

$$
\|d \hbar\|_{p, O} \leq C \operatorname{diam}(O)^{-1}\|\hbar-c\|_{p, \rho O}
$$

for all balls or cubes $O$ with $\rho O \subset \Theta$ and all closed forms $c$. Here $1<p<\infty$. 
Lemma 2.3 [11] Let $\hbar$ be a solution of the nonhomogeneous A-harmonic equation (1.4) in a domain $\Theta$ and $0<s, t<\infty$. Then there exists a constant $C$, independent of $\hbar$, such that

$$
\|\hbar\|_{s, O} \leq C|O|^{(t-s) / t s}\|\hbar\|_{t, \rho O}
$$

for all balls $O$ with $\rho O \subset \Theta$, where $\rho>1$ is a constant.

The following definition is introduced in [6].

Definition 2.4 A kernel $K$ on $\mathbb{R}^{n} \times \mathbb{R}^{n}(n \geq 2)$ is said to satisfy the standard estimates if there exist $\alpha, 0<\alpha \leq 1$, and a constant $C$ such that for all distinct points $x$ and $y$ in $\mathbb{R}^{n}$ and all $z$ with $|x-z|<\frac{1}{2}|x-y|$,

$$
\begin{aligned}
& \text { (1) }|K(x, y)| \leq C|x-y|^{-n} \\
& \text { (2) }|K(x, y)-K(z, y)| \leq C\left|\frac{x-z}{x-y}\right|^{\alpha}|x-y|^{-n} \\
& \text { (3) }|K(z, y)-K(x, y)| \leq C\left|\frac{x-z}{x-y}\right|^{\alpha}|x-y|^{-n}
\end{aligned}
$$

The following $L^{p}$-norm and Lipschitz norm inequalities for the composition $G \circ P$ of Green's operator and the potential operator appear in [10].

Lemma 2.5 Let $\hbar \in L^{p}\left(\Theta, \wedge^{\ell}\right), \ell=0,1, \ldots, n, 1<p<\infty$, be a differential form in a bounded convex domain $\Theta \subset \mathbb{R}^{n}, P$ be the potential operator defined in (1.6) with the kernel $K(x, y)$ satisfying the condition (1) of the standard estimates (2.6) and $G$ be Green's operator. Then there exists a constant $C$, independent of $\hbar$, such that

$$
\left\|G(P(\hbar))-(G(P(\hbar)))_{O}\right\|_{p, O} \leq C|O| \operatorname{diam}(O)\|\hbar\|_{p, O}
$$

for all balls $O$ with $O \subset \Theta$.

Lemma 2.6 Let $\hbar \in L^{p}\left(\Theta, \wedge^{\ell}\right), \ell=0,1, \ldots, n, 1<p<\infty$, be a differential form in a bounded domain $\Theta, P$ be the potential operator defined in (1.6) with the kernel $K(x, y)$ satisfying the condition (1) of the standard estimates (2.6) and G be Green's operator. Then there exists a constant $C$, independent of $\hbar$, such that

$$
\|G(P(\hbar))\|_{\operatorname{locLip}_{k}, \Theta} \leq C\|\hbar\|_{p, \Theta},
$$

where $k$ is a constant with $0 \leq k \leq 1$.

Lemma 2.7 [13] Let $\varphi$ be a strictly increasing convex function on $[0, \infty)$ with $\varphi(0)=0$ (that is, $\varphi$ is a Young function), and $D$ be a bounded domain in $\mathbb{R}^{n}$. Assume that $\hbar$ is a smooth differential form in $D$ such that $\varphi\left(k\left(|\hbar|+\left|\hbar_{D}\right|\right)\right) \in L^{1}(D ; \mu)$ for any real number $k>0$ and $\mu\left(\left\{x \in D:\left|\hbar-\hbar_{D}\right|>0\right\}\right)>0$, where $\mu$ is a Radon measure defined by $d \mu=w(x) d x$ for a weight $w(x)$. Then, for any positive constant $a$, we have

$$
\int_{D} \varphi(a|\hbar|) d \mu \leq C \int_{D} \varphi\left(2 a\left|\hbar-\hbar_{D}\right|\right) d \mu,
$$

where $C$ is a positive constant. 
Using Lemma 2.7 with $\varphi(t)=t^{p}$ and $w(x)=1$ over the ball $O$, we obtain

$$
\|\hbar\|_{p, O} \leq C\left\|\hbar-\hbar_{O}\right\|_{p, O}
$$

where $C$ is a constant.

Theorem 2.8 Let $\hbar \in L^{p}\left(\Theta, \wedge^{\ell}\right), \ell=0,1, \ldots, n, 1<p<\infty$, be a solution of the nonhomogeneous A-harmonic equation (1.4) in a bounded convex domain $\Theta, P$ be the potential operator defined in (1.6) with the kernel $K(x, y)$ satisfying the condition (1) of the standard estimates (2.6) and $G$ be Green's operator. Then there exists a constant $C$, independent of $\hbar$, such that

$$
\|G(P(\hbar))\|_{\operatorname{locLip}_{k}, \Theta} \leq C\|\hbar\|_{\star, \Theta},
$$

where $k$ is a constant with $0 \leq k \leq 1$.

Proof From Lemma 2.5 and (2.10), we obtain

$$
\begin{aligned}
\left\|G(P(\hbar))-(G(P(\hbar)))_{O}\right\|_{p, O} & \leq C_{1}|O| \operatorname{diam}(O)\|\hbar\|_{p, O} \\
& \leq C_{2}|O| \operatorname{diam}(O)\left\|\hbar-\hbar_{O}\right\|_{p, O} .
\end{aligned}
$$

From the decomposition (1.1), (1.2) and (1.3), we have

$$
\left\|\hbar-\hbar_{O}\right\|_{p, O}=\|T d \hbar\|_{p, O} \leq C_{3}|O| \operatorname{diam}(O)\|d \hbar\|_{p, O} \leq C_{4}\left|O\left\|\left.O\right|^{1 / n}\right\| d \hbar \|_{p, O} .\right.
$$

Combining (2.12) with (2.13) yields

$$
\begin{aligned}
\left\|G(P(\hbar))-(G(P(\hbar)))_{O}\right\|_{p, O} & \leq C_{2}|O| \operatorname{diam}(O)\left\|\hbar-\hbar_{O}\right\|_{p, O} \\
& \leq C_{2}|O| \operatorname{diam}(O)\left(C_{4}\left|O\left\|\left.O\right|^{1 / n}\right\| d \hbar \|_{p, O}\right)\right. \\
& \leq C_{5}|O|^{2+1 / n} \operatorname{diam}(O)\|d \hbar\|_{p, O} \\
& \leq C_{6}|O|^{2+2 / n}\|d \hbar\|_{p, O} .
\end{aligned}
$$

Using the definition of the Lipschitz norm, (2.3) with $1=1 / p+(p-1) / p$ and (2.14), for any ball $O$ with $O \subset \Theta$, it follows that

$$
\begin{aligned}
\left\|G(P(\hbar))-(G(P(\hbar)))_{O}\right\|_{1, O} & =\int_{O}\left|G(P(\hbar))-(G(P(\hbar)))_{O}\right| d x \\
& \leq\left(\int_{O}\left|G(P(\hbar))-(G(P(\hbar)))_{O}\right|^{p} d x\right)^{1 / p}\left(\int_{O} 1^{\frac{p}{p-1}} d x\right)^{(p-1) / p} \\
& =|O|^{(p-1) / p}\left\|G(P(\hbar))-(G(P(\hbar)))_{O}\right\|_{p, O} \\
& \leq|O|^{1-1 / p}\left(C_{6}|O|^{2+2 / n}\|d \hbar\|_{p, O}\right) \\
& =C_{6}|O|^{3-1 / p+2 / n}\|d \hbar\|_{p, O}
\end{aligned}
$$


From Lemma 2.2, we have

$$
\|d \hbar\|_{p, O} \leq C_{7} \operatorname{diam}(O)^{-1}\|\hbar-c\|_{p, \rho_{1} O} \leq C_{8}|O|^{-1 / n}\|\hbar-c\|_{p, \rho_{1} O}
$$

for any closed form $c$ and any ball $O$ with $\rho_{1} O \subset \Theta$, where $\rho_{1}>1$ is a constant.

Since $\hbar$ is a solution of equation (1.4) and $c$ is a closed form, $\hbar-c$ is also a solution of equation (1.4). By Lemma 2.3, we obtain

$$
\|\hbar-c\|_{p, \rho_{1} O} \leq C_{9}|O|^{(1-p) / p}\|\hbar-c\|_{1, \rho_{2} O}
$$

for some constant $\rho_{2}>\rho_{1}>1$ with $\rho_{2} O \subset \Theta$.

Combining (2.15), (2.16) and (2.17), we obtain

$$
\begin{aligned}
\left\|G(P(\hbar))-(G(P(\hbar)))_{O}\right\|_{1, O} & \leq C_{6}|O|^{3-1 / p+2 / n}\|d \hbar\|_{p, O} \\
& \leq C_{6}|O|^{3-1 / p+2 / n}\left(C_{8}|O|^{-1 / n}\|\hbar-c\|_{p, \rho_{1} O}\right) \\
& \leq C_{10}|O|^{3-1 / p+1 / n}\|\hbar-c\|_{p, \rho_{1} O} \\
& \leq C_{10}|O|^{3-1 / p+1 / n}\left(C_{9}|O|^{(1-p) / p}\|\hbar-c\|_{1, \rho_{2} O}\right) \\
& \leq C_{11}|O|^{3-1 / p+1 / n+1 / p-1}\|\hbar-c\|_{1, \rho_{2} O} \\
& =C_{11}|O|^{2+1 / n}\|\hbar-c\|_{1, \rho_{2} O}
\end{aligned}
$$

for any closed form $c$.

Since $c$ is any closed form in (2.18), we may choose $c=\hbar_{\rho_{2} O}$ in (2.18). By the definitions of the Lipschitz norms, and noticing $0 \leq k \leq 1$, we find that

$$
\begin{aligned}
\|G(P(\hbar))\|_{\operatorname{locLip}_{k}, \Theta} & =\sup _{\rho_{3} O \subset \Theta}|O|^{-(n+k) / n}\left\|G(P(\hbar))-(G(P(\hbar)))_{O}\right\|_{1, O} \\
& \leq \sup _{\rho_{3} O \subset \Theta}|O|^{-1-k / n}\left(C_{11}|O|^{2+1 / n}\left\|\hbar-\hbar_{\rho_{2} O}\right\|_{1, \rho_{2} O}\right) \\
& =C_{11} \sup _{\rho_{3} O \subset \Theta}|O|^{1+1 / n-k / n}\left\|\hbar-\hbar_{\rho_{2} O}\right\|_{1, \rho_{2} O} \\
& \leq C_{12} \sup _{\rho_{3} O \subset \Theta}|O|^{2+1 / n-k / n}\left|\rho_{2} O\right|^{-1}\left\|\hbar-\hbar_{\rho_{2} O}\right\|_{1, \rho_{2} O} \\
& \leq C_{12} \sup _{\rho_{3} O \subset \Theta}|\Theta|^{2+1 / n-k / n}\left|\rho_{2} O\right|^{-1}\left\|\hbar-\hbar_{\rho_{2} O}\right\|_{1, \rho_{2} O} \\
& \leq C_{12}|\Theta|^{2+1 / n-k / n} \sup _{\rho_{3} O \subset \Theta}\left|\rho_{2} O\right|^{-1}\left\|\hbar-\hbar_{\rho_{2} O}\right\|_{1, \rho_{2} O} \\
& \leq C_{13}\|\hbar\|_{\star, \Theta},
\end{aligned}
$$

where $\rho_{3}>\rho_{2}>\rho_{1}$ with $\rho_{3} O \subset \Theta$.

The proof of Theorem 2.8 has been completed.

We have developed some estimates for the Lipschitz norm $\|\cdot\|_{\operatorname{locLip}_{k}, \Theta}$. Now, we establish the following theorem between the Lipschitz norm and the BMO norm. 
Lemma 2.9 [7] If a differential form $\hbar \in \operatorname{locLip}_{k}\left(\Theta, \wedge^{\ell}\right), \ell=0,1, \ldots, n, 0 \leq k \leq 1$, in a bounded convex domain $\Theta$, then $\hbar \in B M O\left(\Theta, \wedge^{\ell}\right)$ and

$$
\|\hbar\|_{\star, \Theta} \leq C\|\hbar\|_{\text {locLip }_{k}, \Theta},
$$

where $C$ is a constant.

Since $G(P(\hbar))$ is a differential form when $\hbar$ is a differential form, we have the following theorem.

Theorem 2.10 If a differential form $G(P(\hbar)) \in \operatorname{locLip}_{k}\left(\Theta, \wedge^{\ell}\right), \ell=1, \ldots, n, 0 \leq k \leq 1$, in a bounded convex domain $\Theta$, then there exists a constant $C$, independent of $\hbar$, such that $G(P(\hbar)) \in B M O\left(\Theta, \wedge^{\ell}\right)$ and

$$
\|G(P(\hbar))\|_{\star, \Theta} \leq C\|G(P(\hbar))\|_{\text {locLip }_{k}, \Theta}
$$

where the definitions of $G$ and $P$ are the same as in the preceding theorem.

Based on the above results, we estimate the BMO norm $\|\cdot\|_{\star, \Theta}$ of composition $G \circ P$ in terms of $L^{p}$ norm.

Theorem 2.11 Let $\hbar \in L^{p}\left(\Theta, \wedge^{\ell}\right), 1<p<\infty$, be a differential form in a bounded convex domain $\Theta, G$ be Green's operator and P be the potential operator defined in equation (1.6) with the kernel $K(x, y)$ satisfying the condition (1) of the standard estimates (2.6). Then there exists a constant $C$, independent of $\hbar$, such that

$$
\|G(P(\hbar))\|_{\star, \Theta} \leq C\|\hbar\|_{p, \Theta} .
$$

Proof From Lemma 2.6, we have

$$
\|G(P(\hbar))\|_{\operatorname{locLip}_{k}, \Theta} \leq C_{1}\|\hbar\|_{p, \Theta} .
$$

Using Theorem 2.10 and (2.23), it follows that

$$
\|G(P(\hbar))\|_{\star, \Theta} \leq C_{2}\|G(P(\hbar))\|_{\operatorname{locLip}_{k}, \Theta} \leq C_{3}\|\hbar\|_{p, \Theta} .
$$

The proof of Theorem 2.11 has been completed.

Similar to the proof of Theorem 2.11, using Theorems 2.8 and 2.10, we can prove the following theorem.

Theorem 2.12 Let $\hbar \in L^{p}\left(\Theta, \wedge^{\ell}\right), 1<p<\infty$, be a solution of the nonhomogeneous A-harmonic equation (1.4) in a bounded convex domain $\Theta, G$ be Green's operator and $P$ be the potential operator defined in equation (1.6) with the kernel $K(x, y)$ satisfying the condition (1) of the standard estimates (2.6). Then there exists a constant $C$, independent of $\hbar$, such that

$$
\|G(P(\hbar))\|_{\star, \Theta} \leq C\|\hbar\|_{\star, \Theta} .
$$




\section{Two weight estimates}

In this section, we discuss the weighted Lipschitz and BMO norms [7]. For $\hbar \in L_{\text {loc }}^{1}(\Theta$, $\left.\wedge^{\ell}, w^{\alpha}\right), \ell=0,1, \ldots, n$, we write $\hbar \in \operatorname{locLip}_{k}\left(\Theta, \wedge^{\ell}, w^{\alpha}\right), 0 \leq k \leq 1$, if

$$
\|\hbar\|_{\text {locLip }_{k}, \Theta, w^{\alpha}}=\sup _{\rho O \subset \Theta}(\mu(O))^{-(n+k) / n}\left\|\hbar-\hbar_{O}\right\|_{1, O, w^{\alpha}}<\infty
$$

for some $\rho>1$, where $\Theta$ is a bounded domain. The measure $\mu$ is defined by $d \mu=w(x)^{\alpha} d x$, $w$ is a weight and $\alpha$ is a real number. For convenience, we will write the simple notation $\operatorname{locLip}_{k}\left(\Theta, \wedge^{\ell}\right)$ for $\operatorname{locLip}_{k}\left(\Theta, \wedge^{\ell}, w^{\alpha}\right)$. Similarly, for $\hbar \in L_{\text {loc }}^{1}\left(\Theta, \wedge^{\ell}, w^{\alpha}\right), \ell=0,1, \ldots, n$, we write $\hbar \in B M O\left(\Theta, \wedge^{\ell}, w^{\alpha}\right)$ if

$$
\|\hbar\|_{\star, \Theta, w^{\alpha}}=\sup _{\rho O \subset \Theta}(\mu(O))^{-1}\left\|\hbar-\hbar_{O}\right\|_{1, O, w^{\alpha}}<\infty
$$

for some $\rho>1$, where the measure $\mu$ is defined by $d \mu=w(x)^{\alpha} d x, w$ is a weight and $\alpha$ is a real number. Again, we will write $B M O\left(\Theta, \wedge^{\ell}\right)$ to replace $B M O\left(\Theta, \wedge^{\ell}, w^{\alpha}\right)$ when it is clear that the integral is weighted.

Definition 3.1 [1] A pair of weights $\left(w_{1}(x), w_{2}(x)\right)$ satisfies the $A_{r, \lambda}(\Theta)$-condition in a set $\Theta \subset \mathbb{R}^{n}$. Write $\left(w_{1}(x), w_{2}(x)\right) \in A_{r, \lambda}(\Theta)$ for some $\lambda \geq 1$ and $1<r<\infty$ with $\frac{1}{r}+\frac{1}{r^{\prime}}=1$ if

$$
\sup _{O \subset \Theta}\left(\frac{1}{|O|} \int_{O} w_{1}^{\lambda} d x\right)^{\frac{1}{\lambda r}}\left(\frac{1}{|O|} \int_{O}\left(\frac{1}{w_{2}}\right)^{\frac{\lambda r^{\prime}}{r}} d x\right)^{\frac{1}{\lambda r^{\prime}}}<\infty .
$$

Lemma 3.2 [10] Let $\hbar \in L^{p}\left(\Theta, \wedge^{\ell}, v\right), \ell=1, \ldots, n, 1<p<\infty$, be a solution of the nonhomogeneous A-harmonic equation (1.4) in a bounded convex domain $\Theta, P$ be the potential operator defined in (1.6) with the kernel $K(x, y)$ satisfying the condition (1) of the standard estimates (2.6) and $G$ be Green's operator. Assume that $\left(w_{1}(x), w_{2}(x)\right) \in A_{r, \lambda}(\Theta)$ for some $\lambda \geq 1$ and $1<r<\infty$. Then there exists a constant $C$, independent of $\hbar$, such that

$$
\left\|G(P(\hbar))-(G(P(\hbar)))_{O}\right\|_{p, O, w_{1}^{\alpha}} \leq C|O| \operatorname{diam}(O)\|\hbar\|_{p, \rho O, w_{2}^{\alpha}}
$$

for all balls $O$ with $\rho O \subset \Theta$, where $\rho>1$ and $\alpha$ are two constants with $0 \leq \alpha<\lambda$.

Theorem 3.3 Let $\hbar \in L^{p}\left(\Theta, \wedge^{\ell}, v\right), \ell=1, \ldots, n, 1<p<\infty$, be a solution of the nonhomogeneous A-harmonic equation (1.4) in a bounded convex domain $\Theta, P$ be the potential operator defined in (1.6) with the kernel $K(x, y)$ satisfying the condition (1) of the standard estimates (2.6) and $G$ be Green's operator. The measures $\mu$ and $\nu$ are defined by $d \mu=w_{1}^{\alpha} d x$, $d \nu=w_{2}^{\alpha} d x$, and $\left(w_{1}(x), w_{2}(x)\right) \in A_{r, \lambda}(\Theta)$ for some $\lambda \geq 1$ and $1<r<\infty$ with $w_{1}(x) \geq \epsilon>0$ for any $x \in \Theta$. Then there exists a constant $C$, independent of $\hbar$, such that

$$
\|G(P(\hbar))\|_{\operatorname{locLip}_{k}, \Theta, w_{1}^{\alpha}} \leq C\|\hbar\|_{p, \Theta, w_{2}^{\alpha}}
$$

where $k$ and $\alpha$ are constants with $0 \leq k \leq 1$ and $0<\alpha \leq 1$.

Proof Since $\mu(O)=\int_{O} w_{1}^{\alpha} d x \geq \int_{O} \epsilon^{\alpha} d x=C_{1}|O|$, we have

$$
\mu(O)^{-1} \leq C_{2}|O|^{-1}
$$


for any ball $O$. Using (3.4) and Lemma 2.1 with $1=1 / p+(p-1) / p$, it follows that

$$
\begin{aligned}
& \left\|G(P(\hbar))-(G(P(\hbar)))_{O}\right\|_{1, O, w_{1}^{\alpha}} \\
& \quad=\int_{O}\left|G(P(\hbar))-(G(P(\hbar)))_{O}\right| d \mu \\
& \quad \leq\left(\int_{O}\left|G(P(\hbar))-(G(P(\hbar)))_{O}\right|^{p} d \mu\right)^{1 / p}\left(\int_{O} 1^{p /(p-1)} d \mu\right)^{(p-1) / p} \\
& =(\mu(O))^{(p-1) / p}\left\|G(P(\hbar))-(G(P(\hbar)))_{O}\right\|_{p, O, w_{1}^{\alpha}} \\
& =(\mu(O))^{1-1 / p}\left\|G(P(\hbar))-(G(P(\hbar)))_{O}\right\|_{p, O, w_{1}^{\alpha}} \\
& \leq(\mu(O))^{1-1 / p}\left(C_{3}|O| \operatorname{diam}(O)\|\hbar\|_{p, \rho O, w_{2}^{\alpha}}\right) \\
& \leq C_{4}(\mu(O))^{1-1 / p}|O|^{1+1 / n}\|\hbar\|_{p, \rho O, w_{2}^{\alpha} .}
\end{aligned}
$$

Notice that $1-1 / p+1 / n-k / n>0$ and $|\Theta|<\infty$, from (3.1), (3.6) and (3.7), we obtain

$$
\begin{aligned}
\|G(P(\hbar))\|_{\operatorname{locLip}_{k}, \Theta, w_{1}^{\alpha}} & =\sup _{\rho O \subset \Theta}(\mu(O))^{-(n+k) / n}\left\|G(P(\hbar))-(G(P(\hbar)))_{O}\right\|_{1, O, w_{1}^{\alpha}} \\
& \leq \sup _{\rho O \subset \Theta}(\mu(O))^{-1-k / n}\left(C_{4}(\mu(O))^{1-1 / p}|O|^{1+1 / n}\|\hbar\|_{p, \rho O, w_{2}^{\alpha}}\right) \\
& =C_{4} \sup _{\rho O \subset \Theta}(\mu(O))^{-1 / p-k / n}|O|^{1+1 / n}\|\hbar\|_{p, \rho O, w_{2}^{\alpha}} \\
& \leq C_{4} \sup _{\rho O \subset \Theta}\left(C_{2}|O|^{-1}\right)^{1 / p+k / n}|O|^{1+1 / n}\|\hbar\|_{p, \rho O, w_{2}^{\alpha}} \\
& \leq C_{5} \sup _{\rho O \subset \Theta}|O|^{1-1 / p+1 / n-k / n}\|\hbar\|_{p, \rho O, w_{2}^{\alpha}} \\
& \leq C_{5} \sup _{\rho O \subset \Theta}|\Theta|^{1-1 / p+1 / n-k / n}\|\hbar\|_{p, \rho O, w_{2}^{\alpha}} \\
& \leq C_{5}|\Theta|^{1-1 / p+1 / n-k / n} \sup _{\rho O \subset \Theta}\|\hbar\|_{p, \rho O, w_{2}^{\alpha}} \\
& \leq C_{6}\|\hbar\|_{p, \Theta, w_{2}^{\alpha}}
\end{aligned}
$$

The proof of Theorem 3.3 has been completed.

We now estimate the $\|\cdot\|_{\star, \Theta, w_{1}^{\alpha}}$ norm in terms of the $L^{p}$-norm.

Theorem 3.4 Let $\hbar \in L^{p}\left(\Theta, \wedge^{\ell}, v\right), \ell=1, \ldots, n, 1<p<\infty$, be a solution of the nonhomogeneous A-harmonic equation (1.4) in a bounded convex domain $\Theta, P$ be the potential operator defined in (1.6) with the kernel $K(x, y)$ satisfying the condition (1) of the standard estimates (2.6) and $G$ be Green's operator. The measures $\mu$ and $v$ are defined by $d \mu=w_{1}^{\alpha} d x$, $d v=w_{2}^{\alpha} d x$, and $\left(w_{1}(x), w_{2}(x)\right) \in A_{r, \lambda}(\Theta)$ for some $\lambda \geq 1$ and $1<r<\infty$ with $w_{1}(x) \geq \epsilon>0$ for any $x \in \Theta$. Then there exists a constant $C$, independent of $\hbar$, such that

$$
\|G(P(\hbar))\|_{\star, \Theta, w_{1}^{\alpha}} \leq C\|\hbar\|_{p, \Theta, w_{2}^{\alpha},}
$$

where $k$ and $\alpha$ are constants with $0 \leq k \leq 1$ and $0<\alpha \leq 1$. 
Proof From (3.1) and (3.2), it follows that

$$
\begin{aligned}
\|\hbar\|_{\star, \Theta, w_{1}^{\alpha}} & =\sup _{\rho O \subset \Theta}(\mu(O))^{-1}\left\|\hbar-\hbar_{O}\right\|_{1, O, w_{1}^{\alpha}} \\
& =\sup _{\rho O \subset \Theta}(\mu(O))^{k / n}(\mu(O))^{-(n+k) / n}\left\|\hbar-\hbar_{O}\right\|_{1, O, w_{1}^{\alpha}} \\
& \leq \sup _{\rho O \subset \Theta}(\mu(\Theta))^{k / n}(\mu(O))^{-(n+k) / n}\left\|\hbar-\hbar_{O}\right\|_{1, O, w_{1}^{\alpha}} \\
& \leq(\mu(\Theta))^{k / n} \sup _{\rho O \subset \Theta}(\mu(O))^{-(n+k) / n}\left\|\hbar-\hbar_{O}\right\|_{1, O, w_{1}^{\alpha}} \\
& \leq C_{1} \sup _{\rho O \subset \Theta}(\mu(O))^{-(n+k) / n}\left\|\hbar-\hbar_{O}\right\|_{1, O, w_{1}^{\alpha}} \\
& \leq C_{1}\|\hbar\|_{\operatorname{locLip}_{k}, \Theta, w_{1}^{\alpha},}
\end{aligned}
$$

where $C_{1}$ is a positive constant. Replacing $\hbar$ by $G(P(\hbar))$ in (3.10), we find that

$$
\|G(P(\hbar))\|_{\star, \Theta, w_{1}^{\alpha}} \leq C_{1}\|G(P(\hbar))\|_{\operatorname{locLip}_{k}, \Theta, w_{1}^{\alpha}}
$$

where $k$ is a constant with $0 \leq k \leq 1$. From Theorem 3.3, we obtain

$$
\|G(P(\hbar))\|_{\operatorname{locLip}_{k}, \Theta, w_{1}^{\alpha}} \leq C_{2}\|\hbar\|_{p, \Theta, w_{2}^{\alpha}}
$$

Substituting (3.12) into (3.11), we have

$$
\|G(P(\hbar))\|_{\star, \Theta, w_{1}^{\alpha}} \leq C_{3}\|\hbar\|_{p, \Theta, w_{2}^{\alpha}} .
$$

We have completed the proof of Theorem 3.4.

\section{Applications}

If we choose $A, B$ to be a special operator, for example, $A(x, d \hbar)=d \hbar|d \hbar|^{s-2}, B=0$, then (1.4) reduces to the following $s$-harmonic equation:

$$
d^{\star}\left(d \hbar|d \hbar|^{s-2}\right)=0
$$

In particular, we may let $s=2$, then (4.1) reduces to

$$
d^{\star}(d \hbar)=0
$$

Moreover, if $\hbar$ is a function ( 0 -form), then equation (4.2) is equivalent to the well-known Laplace's equation $\Delta \hbar=0$. The function $\hbar$ satisfying Laplace's equation is referred to as the harmonic function as well as one of the solutions of equation (4.2). Therefore, all results in Sections 2 and 3 when $\hbar$ is a solution of the nonhomogeneous A-harmonic equation (1.4) still hold for the $\hbar$ that satisfies (4.2). As to the harmonic function, one finds broader applications in the elliptic partial differential equations; see [14] for more related information. 
We may make use of the following two specific examples to conform the convenience of the inequality (2.22) in evaluating the upper bound for the BMO norm of $G(P(\hbar))$. Obviously, we may take advantage of (2.22) to make this estimating process easy, without calculating $\|G(P(\hbar))\|_{\star, \Theta}$ in a complicated way.

Example 4.1 Let $\hbar=\left(x_{2}^{2}+x_{3}^{2}+2 x_{1} x_{2}+2 x_{1} x_{3}+x_{2} x_{3}\right) d x_{1}+\left(x_{1}^{2}+x_{3}^{2}+2 x_{1} x_{2}+2 x_{1} x_{3}+\right.$ $\left.2 x_{2} x_{3}\right) d x_{2}+\left(x_{1}^{2}+x_{2}^{2}+3 x_{1} x_{2}+2 x_{1} x_{3}+2 x_{2} x_{3}\right) d x_{3}, \Theta=\left\{x=\left(x_{1}, x_{2}, x_{3}\right): \mid 0 \leq x_{1}^{2}+x_{2}^{2}+x_{3}^{2}<1\right\}$, $P$ be the potential operator defined in (1.6) with the kernel $K(x, y)$ satisfying the condition (1) of the standard estimates (2.6) and $G$ be Green's operator.

First, by simple computation, we have

$$
\begin{aligned}
& d \hbar=x_{3} d x_{1} \wedge d x_{2}+2 x_{2} d x_{1} \wedge d x_{3}+x_{1} d x_{2} \wedge d x_{3}, \\
& \star(d \hbar)=x_{3} d x_{3}-2 x_{2} d x_{2}+x_{1} d x_{1}, d(\star(d \hbar))=0 .
\end{aligned}
$$

Since $d^{\star}=(-1)^{2 \times 3+1} \star d \star=-\star d \star$,

$$
d^{\star}(d \hbar)=-\star(d(\star(d \hbar)))=0 .
$$

This implies that $\hbar$ satisfies (4.2).

Observe that

$$
\begin{aligned}
\|\hbar\|_{p, \Theta}= & \left(\int_{\Theta}|\hbar|^{p} d x\right)^{1 / p} \\
= & \left(\int _ { \Theta } \left(\left(x_{2}^{2}+x_{3}^{2}+2 x_{1} x_{2}+2 x_{1} x_{3}+x_{2} x_{3}\right)^{2}\right.\right. \\
& +\left(x_{1}^{2}+x_{3}^{2}+2 x_{1} x_{2}+2 x_{1} x_{3}+2 x_{2} x_{3}\right)^{2} \\
& \left.\left.+\left(x_{1}^{2}+x_{2}^{2}+3 x_{1} x_{2}+2 x_{1} x_{3}+2 x_{2} x_{3}\right)^{2}\right)^{p / 2} d x\right)^{1 / p} \\
\leq & \left(\int _ { \Theta } \left((1+1+2+2+1)^{2}\right.\right. \\
& +(1+1+2+2+2)^{2} \\
& \left.\left.+(1+1+3+2+2)^{2}\right)^{p / 2} d x\right)^{1 / p} \\
\leq & \sqrt{194}|\Theta|^{1 / p} \\
= & \sqrt{194}\left(\frac{4 \pi}{3}\right)^{1 / p} .
\end{aligned}
$$

Applying (2.21), we obtain

$$
\|G(P(\hbar))\|_{\star, \Theta} \leq C\|\hbar\|_{p, \Theta} \leq C \sqrt{194}\left(\frac{4 \pi}{3}\right)^{1 / p} .
$$

Example 4.2 Let us assume, in addition to the definitions of $G$ and $P$ of Example 4.1, $\hbar=e^{x_{1}} \sin x_{2}, \Theta=\left\{x=\left(x_{1}, x_{2}\right): \mid 0 \leq x_{1}^{2}+x_{2}^{2}<1\right\}$. 
Similarly, to begin with, we observe that

$$
\begin{aligned}
& \frac{\partial \hbar}{\partial x_{1}}=\frac{\partial^{2} \hbar}{\partial x_{1}^{2}}=e^{x_{1}} \sin x_{2}, \\
& \frac{\partial \hbar}{\partial x_{2}}=e^{x_{1}} \cos x_{2}, \quad \frac{\partial^{2} \hbar}{\partial x_{2}^{2}}=-e^{x_{1}} \sin x_{2} .
\end{aligned}
$$

Thus,

$$
\Delta \hbar=0,
$$

which implies the function $\hbar$ is harmonic.

Observe that

$$
\begin{aligned}
\|\hbar\|_{p, \Theta} & =\left(\int_{\Theta}|\hbar|^{p} d x\right)^{1 / p} \\
& =\left(\int_{0}^{2 \pi} d \theta \int_{0}^{1}\left|e^{r \cos \theta} \sin (r \sin \theta)\right|^{p} r d r\right)^{1 / p} \\
& \leq e^{1}(2 \pi \cdot 1 / 2)^{1 / p} \\
& =\pi^{1 / p} e .
\end{aligned}
$$

Applying (2.21), we obtain

$$
\|G(P(\hbar))\|_{\star, \Theta} \leq C\|\hbar\|_{p, \Theta} \leq C \pi^{1 / p} e .
$$

\section{Competing interests}

The authors declare that they have no competing interests.

\section{Authors' contributions}

ZD finished the proof and the writing work. YX gave ZD some excellent advice on the proof and writing. SD gave ZD lots of help in revising the paper. All authors read and approved the final manuscript.

\section{Author details}

'Department of Mathematics, Harbin Institute of Technology, Harbin, China. ${ }^{2}$ Department of Mathematics, Seattle University, Seattle, WA 98122, USA.

\section{Acknowledgements}

The authors wish to thank the anonymous referees for their time and thoughtful suggestions.

Received: 23 October 2012 Accepted: 8 January 2013 Published: 22 January 2013

\section{References}

1. Agarwal, RP, Ding, S, Nolder, CA: Inequalities for Differential Forms. Springer, New York (2009)

2. Ding, S: Norm estimates for the maximal operator and Green's operator. Dyn. Contin. Discrete Impuls. Syst., Ser. A Math. Anal. 16, $72-78$ (2009)

3. Iwaniec, T, Lutoborski, A: Integral estimates for null Lagrangians. Arch. Ration. Mech. Anal. 125, 25-79 (1993)

4. $\mathrm{Bi}, \mathrm{H}$ : Weighted inequalities for potential operators on differential forms. J. Inequal. Appl. 2010, Article ID 713625 (2010)

5. Martell, JM: Fractional integrals, potential operators and two-weight, weak type norm inequalities on spaces of homogeneous type. J. Math. Anal. Appl. 294, 223-236 (2004)

6. Uribe, DC, Pérez, C: Two-weight, weak-type norm inequalities for fractional integrals, Calderón-Zygmund operators and commutators. Indiana Univ. Math. J. 49(2), 697-721 (2000)

7. Ding, S: Lipschitz and BMO norm inequalities for operators. Nonlinear Anal. 71, 2350-2357 (2009)

8. Scott, $C: L^{p}$-theory of differential forms on manifolds. Trans. Am. Math. Soc. 347, 2075-2096 (1995) 
9. Agarwal, RP, Ding, S: Inequalities for Green's operator applied to the minimizers. J. Inequal. Appl. 2011, Article ID 66 (2011)

10. Dai, Z, Xing, Y, Ding, S, Wang, Y: Inequalities for the composition of Green's operator and the potential operator. J. Inequal. Appl. 2012, Article ID 271 (2012)

11. Nolder, CA: Hardy-Littlewood theorems for A-harmonic tensors. III. J. Math. 43, 613-631 (1999)

12. Li, Y, Shaw, S: A proof of Hölder's inequality using the Cauchy-Schwarz inequality. J. Inequal. Pure Appl. Math. 7(2), Article ID 62 (2006)

13. Xing, Y, Ding, S: Norm comparison inequalities for the composite operator. J. Inequal. Appl. 2009, Article ID 212915 (2009)

14. Gilbarg, D, Trudinger, NS: Elliptic Partial Differential Equations of Second Order. Springer, Berlin (1997)

doi:10.1186/1029-242X-2013-26

Cite this article as: Dai et al.: BMO and Lipschitz norm estimates for the composition of Green's operator and the potential operator. Journal of Inequalities and Applications 2013 2013:26.

Submit your manuscript to a SpringerOpen ${ }^{\circ}$ journal and benefit from:

- Convenient online submission

Rigorous peer review

- Immediate publication on acceptance

- Open access: articles freely available online

- High visibility within the field

- Retaining the copyright to your article

Submit your next manuscript at $\gg$ springeropen.com 